\title{
Evaluation of the Importance of Various Operating and Sludge Property Parameters to the Fouling of Membrane Bioreactors
}

\author{
F. Y. Sun and X. Y. Li* \\ * Department of Civil Engineering, The University of Hong Kong, Pokfulam Road, Hong Kong, China \\ (feiyun@hkusua.hku.hk; xlia@hkusua.hku.hk)
}

\begin{abstract}
A single-fibre microfiltration system was employed to investigate the importance of various operating and sludge property parameters to the membrane fouling during sludge filtration. The sludge was obtained from a submerged membrane bioreactor (SMBR). A series of comparative and correlative filtration and fouling tests were conducted on the influence of the operating variables, sludge properties and the liquid-phase organic substances on the membrane fouling development. The test results were analysed statistically with Pearson's correlation coefficients and the stepwise multi-variable linear regression. According to the statistical evaluation, the membrane fouling rate has a positive correlation with the biopolymer cluster (BPC) concentration, sludge concentration (MLSS), filtration flux and viscosity, a negative correlation with the cross-flow velocity, and a weak correlation with the extracellular polymeric substances (EPS) and soluble microbial products (SMP). BPC appear to be the most important factor to membrane fouling development during the sludge filtration, followed by the filtration flux and MLSS concentration. The cross-flow rate also is important to the fouling control. It is argued that, during membrane filtration of SMBR sludge, BPC interact with sludge flocs at the membrane surface to facilitate the deposition of the sludge cake layer, leading to serious membrane fouling.
\end{abstract}

Keywords Biopolymer clusters (BPC); membrane bioreactor (MBR); membrane fouling; microfiltration; multi-variable linear regression.

\section{INTRODUCTION}

The membrane bioreactor (MBR) has been deemed as one of the most promising processes for wastewater treatment and reuse (Charcosset, 2006). However, the accumulation of biomass and other substances on/in the membrane, a process described generally as membrane fouling (Le-Clech et al., 2006), is still the main constraint to MBR application. In recent years, a wide range of investigations have been carried out on the membrane fouling phenomenon, including the influences of various process variables and parameters on the fouling development. However, due to the complexity and variability of the foulants and operating conditions of the MBR systems, the findings of different studies are often inconsistent with each other. Some researchers believe that the concentrated sludge in the bulk is the major materials to form a fouling layer on the membrane surface and thus, the mixed liquor suspended solids (MLSS) concentration and the related viscosity are the dominant factors for fouling evolution (Yamamoto et al., 1994). However, other researchers consider that the organic substances in MBR sludge suspension, such as soluble microbial products (SMP) and extracellular polymeric substances (EPS), play an essential role in the fouling layer formation (Nagaoka et al., 1996; Huang et al., 2000) Bouhabila et al. (2001) reported that the relative contributions of MLSS, colloids and dissolved matter on membrane fouling were $24 \%, 50 \%$ and 26\%, respectively. However, Wisniewski and Grasmick (1998) argued that more than 52\% of the total resistance of sludge suspension was attributable to the soluble constituents.

In addition to the sludge properties, the MBR operating conditions, such as filtration flux and shear intensity, are also believed to be important to the membrane fouling development (Le-Clech et al., 
2006). A high flux would accelerate membrane fouling and the fluid shear flow can effectively decelerate the fouling layer formation on the membrane surface. Recent research indicates there is a pool of large-size organic substances accumulated in the MBR system (Wang et al., 2006). The large organic assemblages termed as biopolymer clusters (BPC) range from 2.5 to $60 \mu \mathrm{m}$, and they are neither EPS nor SMP. As large organic solutes, BPC are found to play a crucial role in sludge cake deposition and membrane fouling (Wang and Li, 2008, Sun et al., 2008). All of the factors and parameters, including the MBR process variables, sludge properties and the organic contents in the sludge mixture are expected to have various degrees of influences on the membrane fouling process. However, there is still lacking of comprehensive and systematic studies on the relative importance of these factors to the membrane fouling during MBR sludge filtration.

In this study, a single-fibre microfiltration apparatus was developed for the filtration and fouling investigation on the MBR sludge. The effects of various factors and parameters, including the sludge properties (MLSS concentration, EPS and viscosity), operating variables (flux and crossflow velocity) and organic components (SMP and BPC) in the liquid phase of the sludge suspension, on the membrane fouling rate were tested. All of the parameters were varied in certain ranges. The experimental results were analysed with different statistical methods, including Pearson's correlation coefficients and stepwise multi-variables linear regression, to determine the importance of the factors to membrane fouling.

\section{MATERIALS AND METHODS}

\section{MBR sludge and liquid samples}

The activated sludge (AS) was collected from the bulk suspension in a SMBR, which had been in stable operation for more than four years (Wang et al., 2006). The biomass solids of the AS was separated by sedimentation. The AS solids collected was then diluted with $0.05 \% \mathrm{NaCl}$ solution into a series of sludge suspensions of different MLSS concentrations. The large organic substances, or BPC, were obtained from the cake sludge (CS) deposited on the membrane surface of the SMBR. When the membrane was severely fouled, the CS layer was scraped off the membrane using a spatula. The recovered CS was re-suspended and dispersed by stirring it into a $0.05 \% \mathrm{NaCl}$ solution to a SS concentration of around $10 \mathrm{~g} / \mathrm{L}$. The CS suspension was then separated by sedimentation at $4{ }^{\circ} \mathrm{C}$ for $12 \mathrm{hr}$, and the supernatant was collected for organic analysis. A portion of the CS supernatant was filtered through a $0.4-\mu \mathrm{m}$ polycarbonate membrane filter (Osmonics). The organic in the filtrate was regarded as soluble microbial productes (SMP). The difference in organic content between the CS supernatant and its filtrate was the BPC in the CS. The CS supernatant with a high $\mathrm{BPC}$ content was dosed at various ratios into the sludge suspensions for the microfiltration tests.

The biomass of the SMBR sludge was also analysed for its EPS content. A sludge (As or CS) sample of $50 \mathrm{~mL}$ was centrifuged at $4000 \mathrm{~g}$ for $5 \mathrm{~min}$ for sludge-water separation. The sludge pellet in the centrifugal tube was re-suspended using a vortex mixer (Maxi Mix II, Thermolyne) in 0.05\% $\mathrm{NaCl}$ solution to the original volume $(50 \mathrm{~mL})$ of the sludge sample. According to a heat extraction method (Morgan et al., 1990), the sludge suspension was heated to $50^{\circ} \mathrm{C}$ for $25 \mathrm{~min}$ in a water bath. The sludge mixture was then centrifuged at $4000 \mathrm{~g}$ for $15 \mathrm{~min}$, and the centrate was regarded as the EPS extract of the sludge for chemical analysis.

\section{A single-fibre filtration setup and the constant flux microfiltration (MF) test}

A single-fibre filtration apparatus was fabricated for the sludge filtration and fouling tests (Figure 1). The apparatus was made of a plexiglass tube $1.5 \mathrm{~cm}$ in diameter and $50 \mathrm{~cm}$ in height, in which a polyethelene hollow-fibre membrane $\left(0.4 \mu \mathrm{m}\right.$, surface area $=16 \mathrm{~cm}^{2}$, Mitsubishi Rayon) was 
installed. A suction pump and a flow-meter were used to maintain a constant filtration flux mode. The sludge suspension was pumped through the MF test tube in circulation to generate a cross-flow for continuous membrane surface cleaning. The filtration-fouling tests were conducted using the single-fibre MF system on the sludge samples at different filtration fluxes and different recirculation rates. The membrane fouling rate was measured by the increase in trans-membrane pressure (TMP) with the amount of permeate produced (filtrate depth, L), i.e., $\Delta \mathrm{TMP} / \Delta \mathrm{L}$. Before each test on a sludge suspension, the SMP, EPS and viscosity of the sludge were measured.

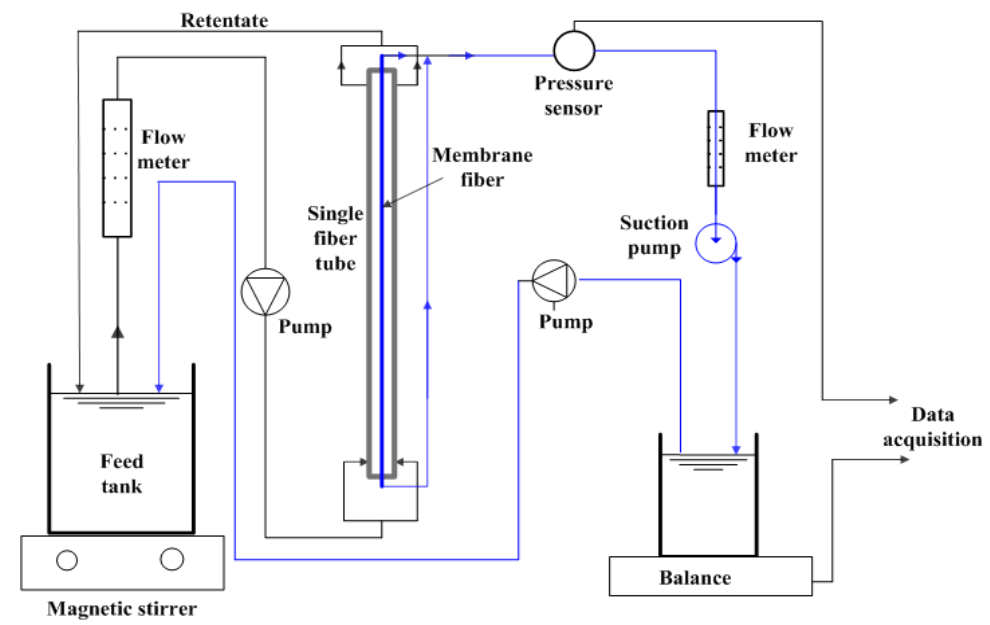

Figure 1. Schematic of the single-fibre microfiltration testing system.

\section{Analytical analysis}

All of the liquid samples, including the SMBR supernatant, the AS supernatant and its filtrate, the CS supernatant and its filtrate, and the EPS extract of the sludge, were analysed for their organic content in terms of the total organic carbon (TOC) concentration. The BPC content in CS was calculated from the difference of TOC in the CS supernatant and its filtrate. The SMP in the AS suspension was determined from the TOC in the AS filtrate. The TOC in a liquid was measured by a TOC analyser (TOC-5000A, Shimadzu), and the viscosity of the sludge suspension was determined by a vibration viscometer (SV-10, A\&D, Japan). The MLSS concentration was measured following the Standard Methods (APHA-AWWA-WEF, 1998).

\section{Statistical analysis of the importance of various factors to membrane fouling}

Based on the results of the MF fouling tests, the importance of a number of factors and parameters to membrane fouling was evaluated, including the operational variables (filtration flux and crossflow rate), sludge property parameters (MLSS, viscosity and EPS) and the organic substances in the sludge suspension (BPC and SMP). The ranges of the parameters and variables tests are summarised in Table 1. With different combinations of the values of the parameters, more than 30 sludge filtration-fouling tests were conducted. The statistical analysis of the fouling test results was performed using the SPSS 13.0 software (SPSS, Chicago, IL, USA) for Windows. For analysis of the importance of the factors to membrane fouling, the process and sludge parameters were tested as predictors (independent factors) and the specific fouling rate $(\Delta \mathrm{TMP} / \Delta \mathrm{L})$ was deemed as the dependent variable. Three different statistical techniques were selected for analysis of the test results. Pearson correlation analysis was used to screen the highly correlated variables from the ranking of the correlative coefficients. Principal component analysis (PCA) was conducted to model the membrane fouling rate from the independent variables. The stepwise least squares multivariable linear regression (MLR) method, with the fouling rate as the dependent variable, was employed to assess the relationship between the fouling rate and various independent variables. Final models were selected when every variable in the model was significant and none of the 
variables outside the model had a significant effect on the prediction.

Table 1. Ranges of the variables and parameters tested during the sludge filtration experiments

\begin{tabular}{ll||ll}
\hline Factor & Test range & Factor & Test range \\
\hline Flux $\left(\mathrm{L} / \mathrm{m}^{2} \cdot \mathrm{hr}\right)$ & $17.5-135$ & Viscosity $(\mathrm{mPa} \cdot \mathrm{s})$ & $1.18-1.98$ \\
Cross-flow rate $(\mathrm{cm} / \mathrm{s})$ & $5.0-75$ & BPC $(\mathrm{mg}$ TOC/L) & $0-10$ \\
MLSS $(\mathrm{g} / \mathrm{L})$ & $1.0-15$ & SMP $(\mathrm{mg}$ TOC/L) & $1.23-5.07$ \\
EPS $(\mathrm{mg} \mathrm{TOC/g} \mathrm{SS)}$ & $10.15-27.60$ & & \\
\hline
\end{tabular}

\section{RESULTS AND DISCUSSION}

\section{Effects of the filtration flux and cross-flow rate on membrane fouling}

The operating condition showed a profound effect on the membrane fouling development. For all of the sludge suspensions tested, the specific fouling rate $(\Delta \mathrm{TMP} / \Delta \mathrm{L})$ increased significantly with an increase in the filtration flux and decreased considerably with an increase in the recirculation flowrate. One example is given in Figure 2 for the test on the sludge having a MLSS of $3 \mathrm{~g} / \mathrm{L}$.

With an increase in permeate flux through the membrane, the drag force increased which would force more foulants in the sludge suspension, such as biomass flocs and organic matter, to deposit on the membrane surface ( $\mathrm{Li}$ and Wang, 2006). The sludge layer formation on the membrane would result in rapid membrane fouling. To the contrary, an increase in the recirculation rate increased the cross-flow velocity over the membrane surface. This would enhance the removal of sludge from the membrane surface ( $\mathrm{Li}$ and Wang, 2006). Hence, a faster cross-flow velocity would reduce the membrane fouling rate effectively.

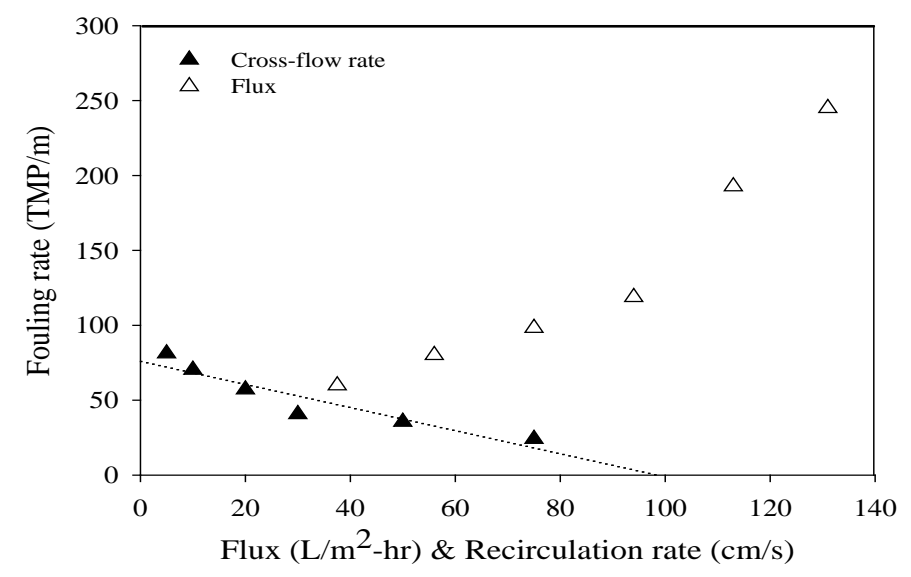

Figure 2. Effects of the permeate flux and cross-flow velocity on membrane fouling.

\section{Influences of the sludge properties on membrane fouling}

All of the three sludge property parameters tested, MLSS concentration, microbial EPS and the liquid viscosity, showed a positive impact on the membrane fouling. As an example, the effects of the sludge properties on the specific TMP increase rate are given in Figure 3(a) for a filtration condition with a moderate flux of $75 \mathrm{~L} / \mathrm{m}^{2} \cdot \mathrm{hr}$ and a cross-flow velocity of $18 \mathrm{~cm} / \mathrm{s}$. It has been recognised that the solids deposition rate onto the membrane surface increases nearly linearly with the MLSS concentration ( $\mathrm{Li}$ and Wang, 2006). Thus, a higher sludge concentration would result in faster sludge cake formation and hence a rapid fouling development.

Viscosity is a parameter representing the rheologic behaviour of the liquor. The viscosity of the 
sludge may be used as a measure of the liquid friction or the stickiness of the organic matter in the liquid. Many researchers have found that there is a strong dependency of the membrane filtration resistance on the viscosity (Nagaoka, 1999; Meng et al., 2006), which is in well agreement with the filtration theory, e.g., Darcy's law. A high viscosity is believed to correlate well with the MLSS concentration and the EPS content of the sludge (Song and Elimelech, 1995). EPS are the construction materials for microbial aggregates and provide a hydrated gel matrix in which microorganisms are embedded. Due to their expected stickiness, EPS are believed to have a negative impact on the filterability of MBR sludge (Nagaoka et al., 1996; Meng et al., 2006). A higher EPS content would facilitate the sludge deposition on the membrane surface, forming a sludge fouling layer with a higher filtration resistance.
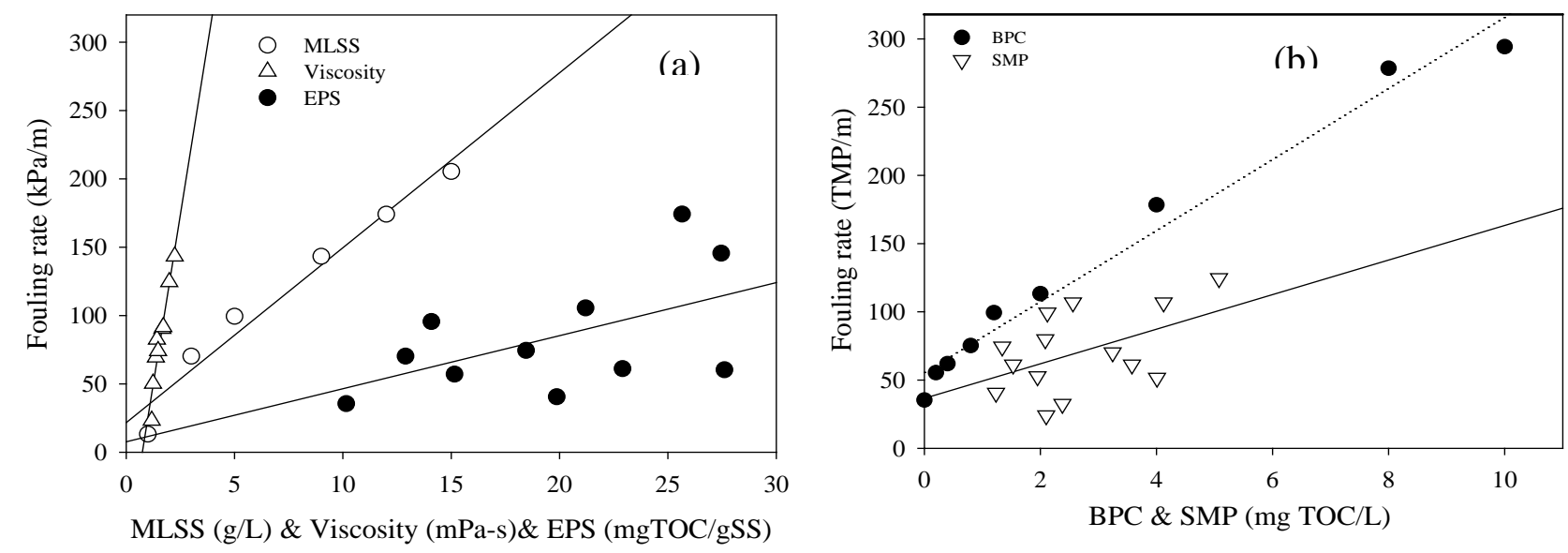

Figure 3. Effects of the sludge properties and organic matter on the membrane fouling rate: (a) sludge properties: MLSS, viscosity and EPS; (b) organic contents: SMP and BPC.

\section{Influences of the organic matter in sludge suspension on membrane fouling}

Organic constitutes in the sludge suspension apparently played an important role in the membrane fouling development. As shown in Figure 3(b), for a given filtration condition, the specific fouling rate increased almost linearly with the BPC content $\left(\mathrm{R}^{2}=0.937\right)$ and had a positive correlation with the SMP concentration. The importance of SMP to membrane fouling has been suggested by previous studies (Huang et al., 2000; Liang et al., 2007). However, due to their soluble and filterable feature, SMP are not expected to significantly affect the filterability of the sludge. In actuality, the MF fouling test results exhibited only a weak correlation $\left(R^{2}=0.238\right)$ between the membrane fouling rate and the SMP concentration of the sludge suspension. In comparison, BPC had an excellent linear correlation with the membrane fouling rate, implying that a high BPC content in sludge mixture would dramatically increase the TMP during membrane filtration. It has been indicated that BPC are an important foulant in SMBR which interact with sludge flocs to facilitate the attachment of sticky and impermeable sludge cake on the membrane surface, resulting in serious membrane fouling (Wang and Li, 2008; Sun et al., 2008).

\section{Multi-variable analysis of the factors on membrane fouling}

As all of the parameters showed various degrees of impacts on the fouling rate, statistical analysis was conducted to determine the importance of the factors to membrane fouling. The result of Pearson's correlation coefficients indicates that BPC, flux, MLSS, cross-flow and viscosity are the key factors that affect membrane fouling (Table 2). In comparison, SMP and EPS are less important to the fouling development. Meanwhile, SMP show a positive correlation with the MLSS and EPS content, which agrees well with the previous studies (Liang et al., 2007). The fouling rate correlates negatively with the cross-flow velocity, and BPC is the most important parameter causing 
membrane fouling. It is also found that viscosity has a positive dependence on the MLSS and BPC concentrations in the sludge suspension. Thus, according to the analysis of Pearson's correlation coefficients, the most important independent variables affecting membrane fouling are in a rank of BPC, filtration flux, cross-flow velocity and MLSS.

Table 2. Pearson's correlation coefficients between the independent parameters and dependent variable.

\begin{tabular}{llllllll}
\hline & $\begin{array}{l}\text { Flux } \\
\left(\mathrm{L} / \mathrm{m}^{2} \cdot \mathrm{hr}\right)\end{array}$ & $\begin{array}{l}\text { Cross-flow } \\
\text { rate }(\mathrm{cm} / \mathrm{s})\end{array}$ & $\begin{array}{l}\text { MLSS } \\
(\mathrm{g} / \mathrm{L})\end{array}$ & $\begin{array}{l}\text { EPS } \\
(\mathrm{mg} \text { TOC/g SS })\end{array}$ & $\begin{array}{l}\text { BPC } \\
(\mathrm{mg} \text { TOC/L) }\end{array}$ & $\begin{array}{l}\text { SMP } \\
(\mathrm{mg} \text { TOC/L) }\end{array}$ & $\begin{array}{l}\text { Viscosity } \\
(\mathrm{mPa} \cdot \mathrm{s})\end{array}$ \\
\hline $\begin{array}{l}\text { Fouling rate } \\
(\mathrm{kPa} / \mathrm{m})\end{array}$ & $\mathbf{0 . 4 3 4}$ & \multirow{2}{*}{$\mathbf{0 . 3 2 7}$} & $\mathbf{0 . 2 4 0}$ & 0.084 & $\mathbf{0 . 8 7 1}$ & 0.126 & $\mathbf{0 . 7 6 6}$ \\
$\mathrm{MLSS}$ & & & & 0.188 & 0.132 & $\mathbf{0 . 3 1 0}$ & $\mathbf{0 . 4 9 9}$ \\
$\mathrm{BPC}$ & & & & & 0.026 & 0.140 & 0.313 \\
EPS & & & & & & 0.219 & 0.276 \\
\hline
\end{tabular}

The results of the stepwise multi-variable linear regression (MLR) of the factors on the membrane fouling rate are given in Table 3. Based on the progress from Model 1 to Model 5, it can be determined that BPC, cross-flow velocity, filtration flux and MLSS are the principal parameters that regulate the fouling rate. Accordingly, the following equation can be obtained,

$$
\frac{\Delta T M P}{\Delta L}=1.497[\text { flux }]-3.731[\text { flowrate }]+10.84[\text { MLSS }]+19.598[\text { BPC }]
$$

where the units of the parameters are the same as those in Table 2. The fouling rate predicted by equation 1 compares well with the experimental measurement (Figure 4 ), as the regression has a $\mathrm{R}^{2}$ value of 0.937 at the $90 \%$ significance level $\left(\mathrm{F}_{\mathrm{Exp}}>\mathrm{F}_{\text {cal }}\right)$. According to the MLR results, BPC apparently is the most important parameter, followed by MLSS, cross-flow rate and then flux, to cause the TMP increase during SMBR sludge filtration.

Table 3. Stepwise MLR results for modelling the MF membrane fouling rate.

\begin{tabular}{lllll}
\hline Models & Predictors & $\mathrm{R}^{2}$ & Adjusted $\mathrm{R}^{2}$ & $\begin{array}{l}\text { Std. Error of } \\
\text { the Estimate }\end{array}$ \\
\hline 1 & Viscosity & 0.587 & 0.579 & 87.35409 \\
2 & Viscosity + BPC & 0.766 & 0.757 & 66.38282 \\
3 & Viscosity + BPC + cross flow rate & 0.845 & 0.835 & 54.66648 \\
4 & Viscosity + BPC + cross flow rate + flux & 0.912 & 0.904 & 41.73157 \\
5 & BPC + cross flow rate + flux + MLSS & 0.937 & 0.930 & 35.58737 \\
\hline
\end{tabular}

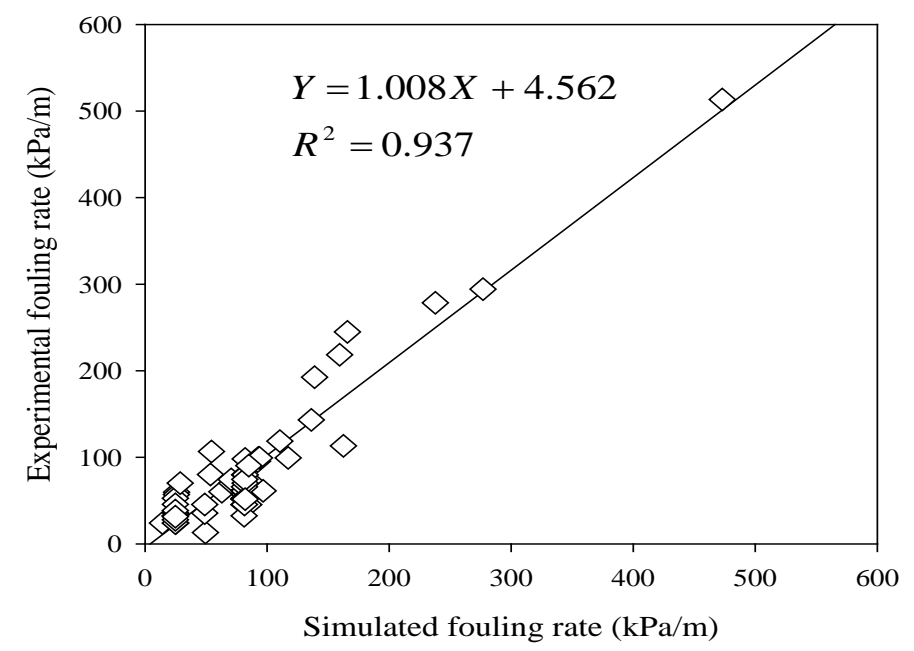

Figure 4. Simulated membrane fouling rate vs. experimental measurement. 


\section{BPC and membrane fouling rate}

Different methods of statistical analysis of the sludge filtration result all indicate the principal importance of BPC to membrane fouling. Meanwhile, sludge concentration and filtration flux are also highly important to the fouling development, whilst the cross-flow velocity can play an essential role in reducing the fouling rate. Viscosity appears to be important; however, it may not be an independent variable in sludge suspension. The fouling test results also suggest that SMP and EPS are not the major foulants in membrane filtration of SMBR sludge.

It has been recognised that BPC are large organic solutes and the major foulants in the SMBR system (Wang et al., 2006; Sun et al., 2008). BPC can function as a glue to facilitate the deposition of biomass sludge on the membrane surface in forming a fouling cake layer. When the BPC dose ratio was low, a cake-dominant layer would be formed. In comparison, when the BPC dose ratio increased to a high level, a gel-layer would form on the membrane surface. In addition, a high BPC content in the sludge deposition can greatly increase the filtration resistance of the cake layer (Wang and $\mathrm{Li}, 2008)$. In the present study, BPC addition in the sludge mixture accelerated the gel-layer formation on the membrane surface together with sludge deposition, resulting in rapid membrane fouling. Moreover, the BPC accumulation in the sludge cake layer would also reduce the effectiveness of cross-flow for membrane cleaning. As BPC had a higher affinity with the membrane and a higher compressibility under pressure, the BPC assemblage would have a higher resistance to the removal by fluid shear from the membrane surface compared to AS flocs.

It should be noted that, although SMP and EPS are not the major and direct foulants during sludge filtration, they are supposed to be the main precursors of BPC in the SMBR sludge mixture (Wang and Li, 2008; Sun et al., 2008). SMP consist to a great extent of hydrophobic substances that can be assembled to large sizes during membrane filtration. The affinity clustering of SMP and loose EPS at the membrane surface would result in considerable formation of BPC within the SMBR system (Sun et al., 2008). During membrane filtration, sticky BPC interact with sludge matters at the membrane surface to build up a cake layer, leading to a serious fouling development.

\section{CONCLUSIONS}

In this study, a single-fibre MF membrane filtration apparatus was developed to evaluate the importance of various process variables and sludge property parameters to membrane fouling during SMBR sludge filtration. A series of comparative filtration and fouling tests were performed, and the results were analysed comprehensively using the statistical techniques. The main findings can be summarised as follows.

1) The membrane fouling rate has a positive correlation with the BPC, MLSS, filtration flux, viscosity, a negative correlation with the cross-flow velocity, and a weak correlation with the EPS and SMP.

2) BPC appear to be the most important foulant to membrane fouling during sludge filtration, followed by the filtration flux and MLSS concentration. The cross-flow can be an effective operating means to control the sludge deposition on the membrane surface.

3) During filtration of SMBR sludge, BPC likely interact with sludge flocs to facilitate the deposition and accumulation of an impermeable sludge cake layer on the membrane surface, leading to progressive membrane fouling.

\section{ACKNOWLEDGEMENTS}

This research was supported by URC funding from the University of Hong Kong, Special Equipment Grant SEG_HKU10 from the University Grants Council (UGC) and Grants HKU7149/E06 and HKU7144/E07 from the Research Grants Council (RGC) of the Hong Kong SAR Government. The technical assistance of Mr. Keith C. H. Wong is greatly appreciated. 


\section{REFERENCES}

APHA-AWWA-WEF, Standard Methods for the Examination of Water and Wastewater, 20th ed., American Public Health Association/American Water Works Association/Water Environment Federation, Washington DC, 1998.

Bouhabila E.H., Aim R.B. \& Buisson H. (2001). Fouling characterization in membrane bioreactors. Separation and Purification Technology, 22/23, 123-132.

Charcosset C. (2006). Membrane processes in biotechnology: an overview. Biotechnology Advance, 24, 482-492.

Gerhardt P., Murray R.G.E., Wood W.A. and Krieg N.R. Methods for general and molecular bacteriology, American Society for Microbiology, Washington, DC, 1994.

Huang X., Liu R. and Qian Y. (2000). Behaviour of soluble microbial products in a membrane bioreactor. Process Biochemistry, 36, 401-406.

Le-Clech P., Chen V. and Fane A.G. (2006). Fouling in membrane bioreactors used in wastewater treatment. Journal of Membrane Science, 284, 17-53.

Li X.Y. and Wang X.M. (2006). Modelling of membrane fouling in a submerged membrane bioreactor. Journal of Membrane Science, 278, 151-161.

Liang S., Liu C. and Song L. (2007). Soluble microbial products in membrane bioreactor operation: Behaviors, characteristics, and fouling potential. Water Research, 41, 95-101.

Meng F., Zhang H., Yang F., Zhang S., Li Y. and Zhang X. (2006). Identification of activated sludge properties affecting membrane fouling in submerged membrane bioreactors. Separation and Purification Technology, 51, 95103.

Morgan J.W., Forster C.F. and Evison L. (1990). A comparative-study of the nature of biopolymers extracted from anaerobic and activated sludge. Water Research, 24, 743-750.

Nagaoka H. (1999). Nitrogen removal by submerged membrane separation activated sludge process. Water Science and Technology, 39(8), 107-114.

Nagaoka H., Ueda S. and Miya A. (1996). Influence of bacterial extracellular polymers on the membrane separation activated sludge process. Water Science and Technology, 34(9), 165-172.

Song L. and Elimelech M. (1995). Theory of concentration polarization in crossflow filtration. Journal of the Chemical Society, Faraday Transactions, 91(19), 3389-3398.

Sun F.Y., Wang X.M. and Li X.Y. (2008). Visualisation and characterisation of biopolymer clusters in a submerged membrane bioreactor. Journal of Membrane Science, 325, 691-697.

Wang X.M. and Li X.Y. (2008). Accumulation of biopolymer clusters in a submerged membrane bioreactor and its effect on membrane fouling. Water Research, 42, 855-862.

Wang X.M., Li X.Y. and Huang X. (2007). Membrane fouling in a submerged membrane bioreactor (SMBR): characterisation of the sludge cake and its high filtration. Separation and Purification Technology, 52, 439-445.

Wisniewski C. and Grasmick A. (1998). Floc size distribution in a membrane bioreactor and consequences for membrane fouling. Colloids Surface A: Physicochemical Engineering Aspects, 138, 403-411.

Yamamoto K., Hissa M., Mahmood T. and Matsuo T. (1994). Direct solid liquid separation using hollow fiber membrane in an activated sludge aeration tank. Water Science and Technology, 30(4), 21-27. 